Volume 70, Number 2, Pages 690-701(2021)

DOI: $10.31801 /$ cfsuasmas.727771

ISSN 1303-5991 E-ISSN 2618-6470

Received by the editors: April 27, 2020; Accepted: March 8, 2021

\title{
SOME FIXED POINT THEOREMS ON COMPLEX VALUED MODULAR METRIC SPACES WITH AN APPLICATION
}

\author{
Kübra ÖZKAN ${ }^{1}$, Utku GÜRDAL ${ }^{2}$, and Ali MUTLU ${ }^{1}$ \\ ${ }^{1}$ Manisa Celal Bayar University, Faculty of Science and Arts, Department of Mathematics, \\ 45140 Manisa, TURKEY \\ ${ }^{2}$ Burdur Mehmet Akif Ersoy University, Faculty of Science and Arts, Department of \\ Mathematics, 15030 Burdur, TURKEY
}

\begin{abstract}
In this article, we introduce the notion of complex valued modular metric spaces. We also a prove generalization of Banach Fixed Point Theorem, which is one of the most simple and significant tests for existence and uniqueness of solution of problems arising in mathematics and engineering for complex valued modular metric spaces. In addition, we express some results related to these spaces. Finally, we give an application of our results to digital programming.
\end{abstract}

\section{INTRODUCTION AND PRELIMINARIES}

In 2011, Azam et al. 6 introduced the notion of complex valued metric spaces and they gave generalization of Banach contraction mapping principle 10]. Then, this space has been studied by many authors. After that, they obtained various fixed point theorems on this spaces $2,7,7,15,16,22,24,31,32,33,34$. On the other hand, a lot of researchers have contributed introducing different concepts on these structures. And they extended them to b-metric, rectangular metric, generalized metric spaces, etc. [1,4,5, 20, 25, 26, 35].

In 1950, Nakano introduced modular spaces 30. In 2008, Chistyakov introduced the notion of modular metric spaces, which has a physical interpretation 11 and he gave the fundamental properties of modular metric spaces 12. In 2011, Mongkolkeha and et. al. proved contraction-type fixed point theorems on modular metric spaces 23. Since the 2010, many researchers as Kumam, Cho, Alaca,

2020 Mathematics Subject Classification. 46A80, 47H10, 54H25.

Keywords and phrases. Fixed point, complex valued modular metric spaces, completeness.

\kubra.ozkan@hotmail.com-Corresponding author; utkugurdal@gmail.com; abgamutlu@gmail.com

(D) 0000-0002-8014-1713; 0000-0003-2887-2188; 0000-0002-6963-4381. 
Khamsi, Mutlu have contributed to develop these structures introducing various fixed point theorems on modular metric spaces $[9,3,8,13,14,17,18,19,27,28$.

The aim of this paper is to introduced the concept of complex valued modular metric spaces, which is more general than well-know modular metric spaces, and give some fixed point theorems under the contraction condition in these spaces. Further, we discuss some results and an application related to these new spaces in digital programming.

Complex valued modular metric spaces form a special class of cone modular metric space. This idea is useful in defining rational expressions which are not meaningful in cone modular metric spaces. Thus, many results of analysis cannot be generalized to cone modular metric spaces. So the complex valued modular metric spaces are important spaces.

Let $z_{1}, z_{2} \in \mathbb{C}, z_{1}=a_{1}+i b_{1}, z_{2}=a_{2}+i b_{2}$ where $a_{1}, b_{2}, a_{1}, b_{2} \in \mathbb{R}$ and $\precsim$ be a partial order on $\mathbb{C}$. Then $z_{1} \precsim z_{2} \Leftrightarrow a_{1} \leq a_{2}$ and $b_{1} \leq b_{2}$. Therefore, it is obvious that $z_{1} \precsim z_{2}$, if

(i) $a_{1}=a_{2}$ and $b_{1}=b_{2}$ or;

(ii) $a_{1}<a_{2}$ and $b_{1}=b_{2}$ or;

(iii) $a_{1}=a_{2}$ and $b_{1}<b_{2}$ or;

(iv) $a_{1}<a_{2}$ and $b_{1}<b_{2}$.

Specially, $z_{1} \lesssim z_{2}$ if $z_{1} \neq z_{2}$ and one of conditions (ii), (iii), (iv) is satisfied. Also, $z_{1} \prec z_{2}$ if only the condition (iv) is satisfied.

Definition 1. [29] Let $X$ be a linear space on $\mathbb{R}$ (or $\mathbb{C}$ ). If a functional $\rho: X \rightarrow$ $[0, \infty]$ holds the following conditions, we call that $\phi$ is a modular on $X:(1) \rho(0)=0$;

(2) If $x \in X$ and $\rho(\alpha x)=0$ some numbers $\alpha>0$, then $x=0$;

(3) $\rho(-x)=\rho(x)$, for all $x \in X$;

(4) $\rho(\alpha x+\beta y) \leq \rho(x)+\rho(y)$ for some $\alpha, \beta \geq 0$ with $\alpha+\beta=1$ and $x, y \in X$.

\section{MAIN RESULTS}

Let $X \neq \emptyset, \lambda \in(0, \infty)$ and $\omega:(0, \infty) \times X \times X \rightarrow \mathbb{C}$ is a function. Throughout this article, the value $\omega(\lambda, x, y)$ is denoted as $\omega_{\lambda}(x, y)$ for all $\lambda>0$ and $x, y \in X$.

Definition 2. Let $X \neq \emptyset$. The function $\omega:(0, \infty) \times X \times X \rightarrow \mathbb{C}$ is called a complex valued metric modular on $X$, if

(CM1) $\omega_{\lambda}(x, y)=0 \Leftrightarrow x=y$;

(CM2) $\omega_{\lambda}(x, y)=\omega_{\lambda}(y, x)$;

(CM3) $\omega_{\lambda+\mu}(x, y) \precsim \omega_{\lambda}(x, z)+\omega_{\mu}(z, y)$

for all $x, y, z \in X$ and $\lambda, \mu>0$.

If instead of (CM1), we only have the condition

$\left(\boldsymbol{C M} 1^{*}\right) \omega_{\lambda}(x, x)=0$ for all $\lambda>0, x \in X$,

then $\lambda$ is said to be a complex valued metric pseudo-modular on $X$. 
Definition 3. Let $\omega:(0, \infty) \times X \times X \rightarrow \mathbb{C}$ be a complex valued metric (pseudo-) modular on $X$. For any $x_{0} \in X$, the sets

$$
X_{\omega}=\left\{x \in X: \lim _{\lambda \rightarrow \infty} \omega_{\lambda}\left(x, x_{0}\right)=0\right\}
$$

and

$$
X_{\omega}^{*}=\left\{x \in X: \exists \lambda=\lambda(x)>0 \text { such that } \omega_{\lambda}\left(x, x_{0}\right)<+\infty\right\}
$$

are said to be complex valued modular spaces (around $x_{0}$ ).

If $\omega$ is complex valued metric modular on $X$, the complex valued modular spaces $X_{\omega}$ can be equipped with a metric, generated by $\omega$ and given by

$$
d_{\omega}(x, y)=\inf \left\{\lambda>0: \omega_{\lambda}(x, y) \precsim \lambda\right\} \text { for any } x, y \in X_{\omega} .
$$

Example 4. Let $(X, d)$ be a complex valued metric space. Then the functional $\omega:(0, \infty) \times X \times X \rightarrow \mathbb{C}$ defined by

$$
\omega_{\lambda}(x, y)=\frac{d(x, y)}{\lambda}
$$

is a complex valued modular metric on $X$. Indeed, complex valued metric spaces are also complex valued modular metric spaces.

Definition 5. Let $X_{\omega}$ be a complex valued modular metric space and $\left\{a_{n}\right\}_{n \in \mathbb{N}}$ be a sequence on $X_{\omega}$. Then,

(1) $\left\{a_{n}\right\}_{n \in \mathbb{N}}$ is called a complex valued modular convergent sequence to a $\in X_{\omega}$, if for every $\epsilon \in \mathbb{C}$ with $\epsilon \succ 0$ there exists $n_{0} \in \mathbb{N}$ such that $\omega_{\lambda}\left(a_{n}, a\right) \prec \epsilon$ for all $\lambda>0$ and $n \geq n_{0}$. And this is denoted with $a_{n} \rightarrow a$ as $n \rightarrow \infty$ or $\lim _{n \rightarrow \infty} a_{n}=a$.

(2) $\left\{a_{n}\right\}_{n \in \mathbb{N}}$ is called a complex valued modular Cauchy sequence, if for every $\epsilon \in \mathbb{C}$ with $\epsilon \succ 0$ there exists $n_{0} \in \mathbb{N}$ such that $\omega_{\lambda}\left(a_{n}, a_{n+m}\right) \prec \epsilon$ for all $\lambda>0$ and $n \geq n_{0}$ as $m \in \mathbb{N}$. This is denoted with $\lim _{n \rightarrow \infty} \omega_{\lambda}\left(a_{n}, a_{n+m}\right)=0$ for all $\lambda>0$ and $m \succ 0$.

(3) $X_{\omega}$ is called a complete complex valued modular metric space, if every modular Cauchy sequence $\left\{a_{n}\right\}$ on $X_{\omega}$ converges to $a \in X_{\omega}$.

(4) The set $K \subseteq X_{\omega}$ is called closed, if the limit of a complex valued modular convergent sequence on $K$ still in $K$.

(5) The set $K \subseteq X_{\omega}$ is called bounded, if

$$
\delta_{\omega}(K)=\sup \left\{\omega_{\lambda}(x, y) \mid x, y \in K\right\}<\infty
$$

for all $\lambda>0$.

Lemma 6. Let $X_{\omega}$ be a complex valued modular metric space and $\left\{a_{n}\right\}_{n \in \mathbb{N}}$ be a sequence on $X_{\omega}$. Then $\left\{a_{n}\right\}$ converges to $a \in X_{\omega}$ if and only if $\omega_{\lambda}\left(a_{n}, a\right) \rightarrow$ 0 as $n \rightarrow \infty$.

Lemma 7. Let $\omega:(0, \infty) \times X \times X \rightarrow \mathbb{C}$ be a complex valued modular metric space and $\left\{a_{n}\right\}_{n \in \mathbb{N}}$ be a sequence on $X_{\omega}$. Then, $\left\{a_{n}\right\}$ is a complex valued Cauch sequence on $X_{\omega}$ if and only if $\omega_{\lambda}\left(a_{n}, a_{n+m}\right) \rightarrow 0$ as $m, n \rightarrow \infty$ for all $m \in \mathbb{N}$. 
Lemma 8. Let $w$ and $z$ be complex numbers. If $w \succsim 0,|z|<1$ and $w \precsim z w$, then $w=0 \in \mathbb{C}$.

Proof. Let $w=a+i b, z=c+i d$ where $a, b, c, d \in \mathbb{R}$. By properties of complex numbers, we have

$$
w \succsim 0 \Rightarrow a \geq 0, b \geq 0
$$

and

$$
|z|<1 \Rightarrow \sqrt{c^{2}+d^{2}}<1 \Rightarrow\left|c^{2}+d^{2}\right|<1 .
$$

Also, since $z w=(a c-b d)+i(a d+b c), w \precsim z w$ implies

$$
a \leq a c-b d \text { and } b \leq a d+b c .
$$

We assume that $a \neq 0$. Since $a>0$ and $|c| \leq\left|c^{2}+d^{2}\right|<1$, we get $a c<a$. From (2), we have $b d<0$. This implies $b>0$ and $d<0$. Then we obtain that $a d<0$ which contradicts with $b(1-c) \leq a d$ for $|c|<1$. Thus, $a=0$. As $a=0,0<1-c$, from $(2) b(1-c) \leq 0$ and $b=0$. So, $w=a+i b=0 \in \mathbb{C}$.

Theorem 9. Let $X_{\omega}$ be a complete complex valued modular metric space. Suppose that $T: X_{\omega} \rightarrow X_{\omega}$ is a mapping satisfying

$$
\omega_{\lambda}(T x, T y) \precsim z \omega_{\lambda}(x, y), z \in \mathbb{C} \text { as }|z|<1
$$

for all $\lambda>0$ and $x, y \in X_{\omega}$. Then $T$ has a unique fixed point on $X_{\omega}$.

Proof. Let $x_{0} \in X_{\omega}$ be arbitrary. We define a sequence $\left\{x_{n}\right\}$ such that $x_{n+1}=$ $T x_{n}=T^{n} x_{0}$ for all $n \geq 0$. Using (3), we have

$$
\omega_{\lambda}\left(x_{n}, x_{n+1}\right)=\omega_{\lambda}\left(T x_{n-1}, T x_{n}\right) \precsim z \omega_{\lambda}\left(x_{n-1}, x_{n}\right) \precsim \cdots \precsim z^{n} \omega_{\lambda}\left(x_{0}, x_{1}\right)
$$

for $\lambda>0$ and $n \geq 0$.

Using (4) and axiom (iii) in the definition of complex valued metric spaces, we obtain that

$$
\begin{aligned}
\omega_{\lambda}\left(x_{n}, x_{n+s}\right) & \precsim \sum_{j=n}^{n+s-1} \omega_{\frac{\lambda}{s}}\left(x_{j}, x_{j+1}\right) \\
& \precsim \sum_{j=n}^{n+s-1} z^{j} \omega_{\frac{\lambda}{s}}\left(x_{0}, x_{1}\right) \\
& \precsim \frac{z^{n}}{1-z} \omega_{\frac{\lambda}{s}}\left(x_{0}, x_{1}\right)
\end{aligned}
$$

some $\lambda>0, s>0$ and $n \in \mathbb{N}$.

Now, we take limit as $n \rightarrow \infty$,

$$
\begin{aligned}
\lim _{n \rightarrow \infty} \omega_{\lambda}\left(x_{n}, x_{n+s}\right) & \precsim \lim _{n \rightarrow \infty} \frac{z^{n}}{1-z} \omega_{\frac{\lambda}{s}}\left(x_{0}, x_{1}\right) \\
& =\frac{\omega_{\frac{\lambda}{s}}\left(x_{0}, x_{1}\right)}{1-z} \lim _{n \rightarrow \infty} z^{n}
\end{aligned}
$$

We know that $\left|z^{n}\right|=|z|^{n} \rightarrow 0$. Then $z^{n} \rightarrow 0 \in \mathbb{C}$. So, we obtain that

$$
0 \precsim \lim _{n \rightarrow \infty} \omega_{\lambda}\left(x_{n}, x_{n+s}\right)=0 .
$$


for all $\lambda>0$ and $s>0$. From (5), we can say that $\left\{x_{n}\right\}$ is a Cauchy sequence. As $X_{\omega}$ is a complete complex valued modular metric space, there is at least one point $p \in X_{\omega}$ such that $\lim _{n \rightarrow \infty} \omega_{\lambda}\left(x_{n}, p\right)=0$.

We show that $p$ is a fixed point of $T$. By using $(3)$ and the axiom (iii) in the definition of complex valued modular metrics, we get

$$
\begin{aligned}
\omega_{\lambda}(p, T p) & \precsim \omega_{\frac{\lambda}{2}}\left(p, T x_{n}\right)+\omega_{\frac{\lambda}{2}}\left(T x_{n}, T p\right) \\
& \precsim \omega_{\frac{\lambda}{2}}\left(p, x_{n+1}\right)+z \omega_{\frac{\lambda}{2}}\left(x_{n}, p\right)
\end{aligned}
$$

for all $\lambda>0, n \geq 0$ and $z \in \mathbb{C}$ with $|z|<1$. If we take limit as $n \rightarrow \infty$ in (6) for $\lambda>0$ and $z \in \mathbb{C}$, since $x_{n} \rightarrow p$, we obtain that

$$
0 \precsim \lim _{n \rightarrow \infty} \omega_{\lambda}(p, T p) \precsim 0 .
$$

Equation (7) implies $\omega_{\lambda}(p, T p)=0$. So, $T p=p$.

In this sequel of the proof, we show the uniqueness of the fixed point $p$ of the mapping $T$. We assume the existence of a point $r$ which is another fixed point of $T$ as $p \neq r$. From (3), we get

$$
\omega_{\lambda}(p, r)=\omega_{\lambda}(T p, T r) \precsim z \omega_{\lambda}(p, r)
$$

Since $\omega_{\lambda}(p, r), z \in \mathbb{C}$ and $|z|<1$, by Lemma 8, we obtain that $\omega_{\lambda}(p, r)=0$ for all $\lambda>0$. So, $p=r$.

Now, as a corollary of this theorem, we express a generalization of the Banach fixed point principle in complex valued modular metric spaces.

Corollary 10. Let $X_{\omega}$ be a complete complex valued modular metric space, $z$ be a complex number such that Imz $=0$ and $|z|<1$. If $T: X_{\omega} \rightarrow X_{\omega}$ is a mapping satisfying

$$
\omega_{\lambda}(T x, T y) \precsim z \omega_{\lambda}(x, y)
$$

for all $\lambda>0$ and $x, y \in X_{\omega}$, then $T$ has a unique fixed point.

Theorem 11. Let $X_{\omega}$ be a complete complex valued modular metric space. If

$$
\omega_{\lambda}\left(T^{n} x, T^{n} y\right) \precsim z \omega_{\lambda}(x, y)
$$

for all $\lambda>0, n>0, z \in \mathbb{C}$ and $x, y \in X_{\omega}$ as $|z|<1$, then $T$ has a unique fixed point.

Proof. Since

$$
\omega_{\lambda}\left(T^{n} x, T^{n} y\right) \precsim z \omega_{\lambda}(x, y),
$$

from Theorem 9, there exists a unique fixed point $p$ of $T^{n}$ on $X_{\omega}$. Then $T^{n} p=p$ as $p \in X_{\omega}$. Then, we have

$$
T^{n}(T p)=T\left(T^{n} p\right)=T p .
$$

Hence, $T p$ is further fixed point of $T^{n}$. Since $p$ is a unique fixed point of $T^{n}, T p=p$. So, $p$ is a fixed point of the mapping $T$. We assume that there exists another fixed 
point $r$ of $T$. So, $T r=r$. Therefore, $T^{n}(T r)=T r$, which contradicts with the uniqueness of fixed point $p$ for $T^{n}$. Then, $p$ is a unique fixed point of $T$.

Example 12. Let $X=\mathbb{C}$. The mapping $\omega:(0, \infty) \times \mathbb{C} \times \mathbb{C} \rightarrow \mathbb{C}$ is defined by

$$
\omega_{\lambda}\left(z_{1}, z_{2}\right)=\frac{\left|a_{1}-a_{2}\right|+i\left|b_{1}-b_{2}\right|}{\lambda}
$$

for all $\lambda>0$ where $z_{1}=a_{1}+i b_{1}$ and $z_{2}=a_{2}+i b_{2}$. Then, it can be shown that

isacompletecomplexvaluedmodularmetricspace.Wedefineamapping

$T: C_{\omega} \rightarrow \mathbb{C}_{\omega}$ such that $T k=\frac{k}{3}$ and we take $z=\frac{1}{3} \in \mathbb{C}$. Then, for all $z_{1}, z_{2} \in \mathbb{C}$ and $\lambda>0$, we have

$$
\omega_{\lambda}\left(T z_{1}, T z_{2}\right)=\omega_{\lambda}\left(\frac{z_{1}}{3}, \frac{z_{2}}{3}\right)=\frac{\left|a_{1}-a_{2}\right|+i\left|b_{1}-b_{2}\right|}{3 \lambda}
$$

and

$$
\omega_{\lambda}\left(z_{1}, z_{2}\right)=\frac{\left|a_{1}-a_{2}\right|+i\left|b_{1}-b_{2}\right|}{\lambda} .
$$

Hence, $\omega_{\lambda}\left(T z_{1}, T z_{2}\right) \precsim z \omega_{\lambda}\left(z_{1}, z_{2}\right)$. From Theorem 9. $T$ has a fixed point, which is immediately seen to be $0 \in \mathbb{C}$.

Let $X_{\omega}$ be a complex valued modular metric space, $K \subseteq X_{\omega}, \psi: K \rightarrow \mathbb{C}$ be a function and $\left\{x_{n}\right\}$ be a sequence in $K . \psi$ is called lower semi-continuous (l.s.c.) on $K$ if

$$
\lim _{n \rightarrow \infty} \omega_{\lambda}\left(x_{n}, x\right)=0 \text { and } \lim _{n \rightarrow \infty} \inf \left(\psi\left(x_{n}\right)\right)=h \text { imply } \psi(x) \leq h
$$

for all $\left\{x_{n}\right\} \subseteq K$ and $\lambda>0$.

Theorem 13. Let $X_{\omega}$ be a complete complex valued modular metric space and $\psi$ : $X_{\omega} \rightarrow \mathbb{C}$ be a lower semi-continuous function on $X_{\omega}$. If any mapping $T: X_{\omega} \rightarrow X_{\omega}$ satisfying

$$
\omega_{\lambda}(x, T x) \leq \psi(x)-\psi(T x)
$$

for all $\lambda>0$ and $x, y \in X_{\omega}$, then $T$ has a fixed point in $X_{\omega}$.

Proof. For each $x \in X_{\omega}$ denote,

$$
\begin{aligned}
M(x) & =\left\{y \in X_{\omega}: \omega_{\lambda}(x, y) \precsim \psi(x)-\psi(y) \text { for all } \lambda>0\right\}, \\
\alpha(x) & =\inf \{\psi(y): y \in M(x)\} .
\end{aligned}
$$

Let $x \in M(x)$. Then, $M(x)$ is not empty and $0 \leq \alpha(x) \leq \psi(x)$. We take an arbitrary point $x \in X_{\omega}$. Now, we form a sequence $\left\{x_{n}\right\}$ on $X_{\omega}$ as follows:

Let $x_{1}=x$ and when $x_{1}, x_{2}, \ldots, x_{n}$ have been chosen, choose $x_{n+1} \in M\left(x_{n}\right)$ such that

$$
\psi\left(x_{n+1}\right) \leq \alpha\left(x_{n}\right)+\frac{1}{n}
$$

for all $n \geq 1$. By doing so, we get a sequence $\left\{x_{n}\right\}$ satisfying the condition

$$
\begin{aligned}
\omega_{\lambda}\left(x_{n}, x_{n+1}\right) & \precsim \psi\left(x_{n}\right)-\psi\left(x_{n+1}\right), \\
\alpha\left(x_{n}\right) & \leq \psi\left(x_{n+1}\right) \leq \alpha\left(x_{n}\right)+\frac{1}{n}
\end{aligned}
$$


for all $n \geq 0$ and $\lambda>0$. Then, $\left\{\psi\left(x_{n}\right)\right\}$ is a nonincreasing sequence and it is bounded from below by zero. So, the sequence $\left\{\psi\left(x_{n}\right)\right\}$ is convergent to a number $D \geq 0$. By virtue of $(9)$, we get

$$
D=\lim _{n \rightarrow \infty} \psi\left(x_{n}\right)=\lim _{n \rightarrow \infty} \alpha\left(x_{n}\right) .
$$

Now, let $k \in \mathbb{N}$ be arbitrary. From (9) and (10), there exists a number $N_{k}$ such that

$$
\psi\left(x_{n}\right)<D+\frac{1}{k} \text { for all } n \geq N_{k} .
$$

Since $\psi\left(x_{n}\right)$ monotone, we get

$$
D \leq \psi\left(x_{m}\right) \leq \psi\left(x_{n}\right)<D+\frac{1}{k}
$$

for $m \geq n \geq N_{k}$. Then, we obtain that

$$
\psi\left(x_{n}\right)-\psi\left(x_{m}\right)<\frac{1}{k} \text { for all } m \geq n \geq N_{k} .
$$

Preserving the generality, suppose that $m>n$ and $m, n \in \mathbb{N}$. From [11), we get

$$
\omega_{\frac{\lambda}{m-n}}\left(x_{n}, x_{n+1}\right) \precsim \psi\left(x_{n}\right)-\psi\left(x_{n+1}\right)
$$

for all $\frac{\lambda}{m-n}>0$. Now, we obtain that

$$
\begin{aligned}
\omega_{\lambda}\left(x_{n}, x_{m}\right) & \precsim \omega_{\frac{\lambda}{m-n}}\left(x_{n}, x_{n+1}\right)+\omega_{\frac{\lambda}{m-n}}\left(x_{n+1}, x_{n+2}\right)+\cdots+\omega_{\frac{\lambda}{m-n}}\left(x_{m-1}, x_{m}\right) \\
& \precsim \sum_{j=n}^{m-1}\left[\psi\left(x_{j}\right)-\psi\left(x_{j+1}\right)\right] \\
& =\psi\left(x_{n}\right)-\psi\left(x_{m}\right)
\end{aligned}
$$

for all $m, n \geq N_{k}$. Then, by 11,

$$
\omega_{\lambda}\left(x_{n}, x_{m}\right) \prec \frac{1}{k} \quad \text { for all } m \geq n \geq N_{k} .
$$

Letting $k$ or $m, n$ to tend to infinity in $(13)$, we conclude that

$$
\lim _{m, n \rightarrow \infty} \omega_{\lambda}\left(x_{n}, x_{m}\right)=0 .
$$

Then, $\left\{x_{n}\right\}_{n \in \mathbb{N}}$ is a complex valued modular Cauchy sequence. Hence, from the completeness of $X_{\omega}$, there exist a point $p \in X_{\omega}$ such that $x_{n} \rightarrow p$ as $n \rightarrow \infty$. Since $\psi$ is lower semi-continuous, using the equation (12), we have

$$
\begin{aligned}
\psi(p) & \leq \lim _{m \rightarrow \infty} \inf \psi\left(x_{m}\right) \\
& \precsim \lim _{m \rightarrow \infty} \inf \left(\psi\left(x_{n}\right)-\omega_{\lambda}\left(x_{n}, x_{m}\right)\right) \\
& =\psi\left(x_{n}\right)-\omega_{\lambda}\left(x_{n}, p\right)
\end{aligned}
$$

and hence

$$
\omega_{\lambda}\left(x_{n}, p\right) \precsim \psi\left(x_{n}\right)-\psi(p) .
$$


Thus, $p \in M\left(x_{n}\right)$ for all $n \geq 0$ and $\alpha\left(x_{n}\right) \leq \psi(p)$. So, by $[10)$, we have $D \leq \psi(p)$. Moreover, by lower semi-continuity of $\psi$ and $(10)$, we get

$$
\psi(p)=\lim _{n \rightarrow \infty} \psi\left(x_{n}\right)=S .
$$

So, $\psi(p)=S$. From 8 we know that $T p \in M(p)$. Since $p \in M(p)$ for $n \in \mathbb{N}$, we get

$$
\begin{aligned}
\omega_{\lambda}\left(x_{n}, T p\right) & \precsim \omega_{\frac{\lambda}{2}}\left(x_{n}, p\right)+\omega_{\frac{\lambda}{2}}(p, T p) \\
& \precsim \psi\left(x_{n}\right)-\psi(p)+\psi(p)-\psi(T p) \\
& =\psi\left(x_{n}\right)-\psi(T p) .
\end{aligned}
$$

Then $T p \in M\left(x_{n}\right)$ and implies $\alpha\left(x_{n}\right) \leq \psi(T p)$. Thus, we obtain $S \leq \psi(T p)$. Since $\psi(T p) \leq \psi(p)$ by $(8)$ and $\psi(p)=S$, we get

$$
\psi(p)=S \leq \psi(T p) \leq \psi(p) .
$$

Therefore, $\psi(T p)=\psi(p)$. Then from (8), we get

$$
\omega_{\lambda}(p, T p) \precsim \psi(p)-\psi(T p)=0 .
$$

Thus, $T p=p$.

Example 14. Let $X=\mathbb{C}$. We define the mapping $\omega:(0, \infty) \times \mathbb{C} \times \mathbb{C} \rightarrow \mathbb{C}$ by

$$
\omega_{\lambda}\left(z_{1}, z_{2}\right)=\frac{\left|a_{1}-a_{2}\right|+i\left|b_{1}-b_{2}\right|}{\lambda}
$$

for all $\lambda>0$ where $z_{1}=a_{1}+i b_{1}$ and $z_{2}=a_{2}+i b_{2} . \mathbb{C}_{\omega}$ is a complete modular metric space. Define $T: \mathbb{C}_{\omega} \rightarrow \mathbb{C}_{\omega}$ by $T z=\frac{z}{4}$ and $\psi: \mathbb{C}_{\omega} \rightarrow \mathbb{C}$ by $\psi(z)=|a|+i|b|$ where $z=a+i b$. Then for all $z=a+i b \in \mathbb{C}$ and $\lambda>0$, we have

$$
\omega_{\lambda}(z, T z)=\frac{\left|a-\frac{a}{4}\right|+i\left|b-\frac{b}{4}\right|}{\lambda}=\frac{\frac{3}{4}|a|+i \frac{3}{4}|b|}{\lambda} \leq \frac{3}{4}(|a|+i|b|)
$$

and

$$
\psi(z)-\psi(T z)=(|a|+i|b|)-\left(\frac{|a|}{4}+i \frac{|b|}{4}\right)=\frac{3}{4}(|a|+i|b|) .
$$

Hence, $\omega_{\lambda}(z, T z) \leq \psi(z)-\psi(T)$. From Theorem 13, the mapping $T$ has a fixed point.

\section{AN APPLICATION TO DYNAMIC PROGRAMMING}

In the section, we express an application of Theorem 9 to dynamic programming which is a powerful tecnique for solving some complex problems in mathematics, economics, computer science and bioinformatics.

Let $X_{\omega}$ be a complete complex valued modular metric space induced by $\omega$ : $(0, \infty) \times \mathbb{C} \times \mathbb{C} \rightarrow \mathbb{C}, S \subseteq X_{\omega}, Z$ be a Banach space and $P \subseteq Z$.

We consider the functional equation

$$
q(x)=\sup _{y \in P}\{f(x, y)+H(x, y, q(\varphi(x, y)))\}
$$


where $x \in S, \varphi: S \times P \rightarrow S, f: S \times P \rightarrow \mathbb{C}$ and $H: S \times P \times \mathbb{C} \rightarrow \mathbb{C}$. We show that existence of unique solution of the functional equation (14). We suppose that $B(S)$ is the set of all bounded complex valued function on $S$. We define

$$
\|k\|=\sup _{x \in S}|k(x)|
$$

for an arbitrary $k \in B(S)$. We take complex valued metric modular $\omega$ on $B(S)$ as

$$
\omega_{\lambda}(k, g)=\sup _{x \in Z}\left\{\left|\frac{k(x)-g(x)}{\lambda}\right|+i\left|\frac{k(x)-g(x)}{\lambda}\right|\right\}
$$

for all $k, g \in B(S)$ and $\lambda>0$. On the other hand, we take a Cauchy sequence $\left\{k_{n}\right\}_{n \in \mathbb{N}}$ in $B(S)$. Then $\left\{k_{n}\right\}_{n \in \mathbb{N}}$ is convergent to a function $t \in B(S)$.

Theorem 15. Let $f: S \times P \rightarrow \mathbb{C}$ and $H: S \times P \times \mathbb{C} \rightarrow \mathbb{C}$ be bounded. We suppose that $T: B(S) \rightarrow B(S)$ defined by

$$
T(k)(x)=\sup _{y \in P}\{f(x, y)+H(x, y, k(\varphi(x, y)))\}
$$

for all $k \in B(S)$ and $x \in S$. If

$$
\left|\frac{H(x, y, k(x))-H(x, y, g(x))}{\lambda}\right|+i\left|\frac{H(x, y, k(x))-H(x, y, g(x))}{\lambda}\right| \precsim z \omega_{\lambda}(k, g)
$$

for all $\lambda>0, x \in S, y \in P, k, g \in B(S)$ and a arbitrary complex number $z$ where $|z|<1$, the functional equation (14) has a unique solution.

Proof. Let $x \in S$ and $k, g \in B(S)$. Then there exist $y_{1}, y_{2} \in P$ and a complex number $\delta>0$ such that

$$
\begin{aligned}
T(k)(x) & \precsim f\left(x, y_{1}\right)+H\left(x, y_{1}, k\left(\varphi\left(x, y_{1}\right)\right)\right)+\delta \\
T(g)(x) & \precsim f\left(x, y_{2}\right)+H\left(x, y_{2}, g\left(\varphi\left(x, y_{2}\right)\right)\right)+\delta \\
T(k)(x) & \succsim f\left(x, y_{1}\right)+H\left(x, y_{1}, k\left(\varphi\left(x, y_{1}\right)\right)\right) \\
T(g)(x) & \succsim f\left(x, y_{2}\right)+H\left(x, y_{2}, g\left(\varphi\left(x, y_{2}\right)\right)\right) .
\end{aligned}
$$

From (17) and $(20)$, we obtain that

$$
\begin{aligned}
T(k)(x)-T(g)(x) & \precsim \quad H\left(x, y_{1}, k\left(\varphi\left(x, y_{1}\right)\right)\right)-H\left(x, y_{2}, g\left(\varphi\left(x, y_{2}\right)\right)\right)+\delta \\
& \precsim\left|H\left(x, y_{1}, k\left(\varphi\left(x, y_{1}\right)\right)\right)-H\left(x, y_{2}, g\left(\varphi\left(x, y_{2}\right)\right)\right)\right|+\delta .
\end{aligned}
$$

So, for $\lambda>0$

$$
\frac{T(k)(x)-T(g)(x)}{\lambda} \precsim\left|\frac{H\left(x, y_{1}, k\left(\varphi\left(x, y_{1}\right)\right)\right)-H\left(x, y_{2}, g\left(\varphi\left(x, y_{2}\right)\right)\right)}{\lambda}\right|+\frac{\delta}{\lambda} .
$$

Similarly, combining $(18)$ and $(19)$ we have

$$
\frac{T(g)(x)-T(k)(x)}{\lambda} \precsim\left|\frac{H\left(x, y_{1}, k\left(\varphi\left(x, y_{1}\right)\right)\right)-H\left(x, y_{2}, g\left(\varphi\left(x, y_{2}\right)\right)\right)}{\lambda}\right|+\frac{\delta}{\lambda} .
$$


Therefore, from 21) and 222,

$$
\left|\frac{T(k)(x)-T(g)(x)}{\lambda}\right| \precsim\left|\frac{H\left(x, y_{1}, k\left(\varphi\left(x, y_{1}\right)\right)\right)-H\left(x, y_{2}, g\left(\varphi\left(x, y_{2}\right)\right)\right)}{\lambda}\right|+\frac{\delta}{\lambda}
$$

for all $\lambda>0$. Since $\frac{\delta}{\lambda}>0$ in inequality 23), we can ignore the contrary the $\frac{\delta}{\lambda}$. Therefore,

$$
\left|\frac{T(k)(x)-T(g)(x)}{\lambda}\right| \precsim\left|\frac{H\left(x, y_{1}, k\left(\varphi\left(x, y_{1}\right)\right)\right)-H\left(x, y_{2}, g\left(\varphi\left(x, y_{2}\right)\right)\right)}{\lambda}\right| .
$$

From inequality (24), we easily obtain that

$$
\begin{aligned}
\left|\frac{T(k)(x)-T(g)(x)}{\lambda}\right|+i\left|\frac{T(k)(x)-T(g)(x)}{\lambda}\right| \precsim\left|\frac{H\left(x, y_{1}, k\left(\varphi\left(x, y_{1}\right)\right)\right)-H\left(x, y_{2}, g\left(\varphi\left(x, y_{2}\right)\right)\right)}{\lambda}\right| \\
+i\left|\frac{H\left(x, y_{1}, k\left(\varphi\left(x, y_{1}\right)\right)\right)-H\left(x, y_{2}, g\left(\varphi\left(x, y_{2}\right)\right)\right)}{\lambda}\right|
\end{aligned}
$$

From $(15)$ and $(16)$, we get

$$
\omega_{\lambda}(T(k), T(g)) \precsim z \omega_{\lambda}(k, g) .
$$

Then, from Theorem 9. $T$ has a unique fixed point $t \in B(S)$. That is, the functional equation (14) has a unique solution.

Open problem How can we obtain coupled fixed point theorems and common fixed point theorems in these metric spaces?

Authors Contribution Statement The authors contributed equally to this work. All authors of the submitted research paper have directly participated in the planning, execution, or analysis of study.

Declaration of Competing Interests The authors declare that there is no conflict of interest regarding the publication of this article.

\section{REFERENCES}

[1] Abbas, M., Rajic, V. C., Nazir, T., Radenovic, S., Common fixed point of mappings satisfying rational inequalities in ordered complex valued generalized metric spaces, Afrika Matematika, (2013), 1-14. https://doi.org/10.1007/s13370-013-0185-z.

[2] Ahmad, J., Klin-eam, C., Azam, A., Common fixed points for multi-valued mappings in complex valued metric spaces with applications, Abstract and applied analysis, 2013 (2013), Article ID 854965, 12 pages. https://doi.org/10.1155/2013/854965.

[3] Alaca, C., Ege, M.E., Park, C., Fixed point results for modular ultrametric spaces, Journal of Computational Analysis and Applications, 20(7) (2016), 1259-1267.

[4] Ansari, A. H., Ege, Ö., Radenović, S., Some fixed point results on complex valued Gb-metric spaces, Revista de la Real Academia de Ciencias Exactas, Fisicas y Naturales - Serie A: Matematicas, 112 (4), (2017), doi: 10.1007/s13398-017-0391-x.

[5] Asadi, M., Moeini, B., Mukheimer, A., Aydi, H., Complex valued M-metric spaces and related fixed point results via complex C-class function, Journal of Inequalities and Special Functions, 10(1) (2019), 101-110. 
[6] Azam, A., Fisher B., Khan, M., Common fixed point theorems in complex valued metric spaces, Numerical Functional Analysis and Optimization, 32(3) (2011), 243-253. https://doi.org/10.1080/01630563.2011.533046.

[7] Azam, A., Ahmad, J., Kumam, P., Common fixed point theorems for multi-valued mappings in complex-valued metric spaces, Journal of Inequalities and Applications, 2013, 2013:578.

[8] Abdou, A. A. N., Khamsi, M. A., On the fixed points of nonexpansive mappings in modular metric spaces, Fixed Point Theory and Applications, (2013), 2013:229. https://doi.org/10.1186/1029-242X-2013-578.

[9] Aksoy, U., Karapinar, E., Erhan, M., Rakocevic, V., Meir-Keeler type contractions on modular metric spaces, Filomat, 32(10) (2013), 3697-3707. https://doi.org/10.2298/FIL1810697A.

[10] Banach, S., Sur les opérations dans les ensembles abstraits et leur application aux équations intégrales, Fundamenta Mathematicae, 3 (1922), 133-181.

[11] Chistyakov, V. V., Modular metric spaces generated by F-modulars, Folia Mathematica, 14 (2008), 3-25.

[12] Chistyakov, V. V., Modular metric spaces I basic concepts, Nonlinear Analysis, 72 (2010), 1-14. https://doi.org/10.1016/j.na.2009.04.057.

[13] Chistyakov, V. V., Fixed points of modular contractive maps, Doklady Mathematics, 86(1) (2012), 515-518. https://doi.org/10.1134/S1064562412040163.

[14] Cho, Y.J., Saadati, R., Sadeghi, G., Quasi-contractive mappings in modular metric spaces, Journal of Applied Mathematics, (2012), Article ID 907951, 5 pages. https://doi.org/10.1155/2012/907951.

[15] Humaira, H., Sarwar, M., Kumam, P., Common fixed point results for fuzzy mappings on complex-valued metric spaces with homotopy results, Symmetry, 11(1) (2019), 61; https://doi.org/10.3390/sym11010061.

[16] Hussain, N., Ahmad, J., Azam, A., Arshad, M., Common fixed point results in complex valued metric spaces with application to integral equations, Filomat, 28(7) (2014), 1363-1380. https://doi.org/10.2298/FIL1407363H.

[17] Ege, M. E., Alaca, C., Fixed point results and an application to homotopy in modular metric spaces, Journal of Nonlinear Science and Applications, 8(6) (2015), 900-908.

[18] Ege, M. E., Alaca, C., Some properties of modular S-metric spaces and its fixed point results, Journal of Computational Analysis and Applications, 20(1) (2016), 24-33.

[19] Ege, M. E., Alaca, C., Some results for modular b-metric spaces and an application to system of linear equations, Azerbaijan Journal of Mathematics, 8(1) (2018), 3-14.

[20] Ege, Ö., Some fixed point theorems in complex valued $G_{b}$ metric spaces, Journal of Nonlinear and Convex Analysis, 18(11) (2017), 1997-2005.

[21] Kilinç, E., Alaca, C., A Fixed point theorem in modular metric spaces, Advances Fixed Point Theory, 4 (2014), 199-206.

[22] Kutbi, M. A., Azam, A., Ahmad, J., Di Bari, C., Some common coupled fixed point results for generalized contraction in complex-valued metric spaces, Journal of Applied Mathematics, (2013), 2013: Article ID 352927. https://doi.org/10.1155/2013/352927.

[23] Mongkolkeha, C., Sintunavarat, W., Kumam, P., Fixed point theorems for contraction mappings in modular metric spaces, Fixed Point Theory and Applications (2011), 2011:93. https://doi.org/10.1186/1687-1812-2011-93.

[24] Kumam, P., Sarwar, M., Zada, M. B., Fixed point results satisfying rational type contractive conditions in complex valued metric spaces, Annales Mathematicae Silesianae, 30 (2016), 89-110. https://doi.org/10.1515/amsil-2016-0003.

[25] Mukheimer, A., Common fixed point theorems for a pair of mappings in complex valued b-metric spaces, Advances in Fixed Point Theory, 4(3) (2014), 344-354.

[26] Mukheimer, A., Some fixed point theorems in complex valued b-metric spaces, Italian Journal of Pure and Applied Mathematics, 42 (2019), 11-125. 
[27] Mutlu, A., Özkan, K., Gürdal, U., A new fixed point theorem in modular metric spaces, International Journal of Analysis and Applications, 16(4) (2018), 1-10. https://doi.org/10.28924/2291-8639.

[28] Mutlu, A., Özkan, K., Gürdal, U., Coupled fixed point theorem in partially ordered modular metric spaces and its an application, Journal of Computational Analysis and Applications, $25(2)$ (2018), 1-10.

[29] Musielak, J., Orlicz, W., On modular spaces, Studia Mathematica, 18 (1959), 49-65.

[30] Nakano, H., Modulared semi-ordered linear spaces, In Tokyo Math. Book Ser., vol. 1, Maruzen Co., Tokyo, 1950.

[31] Sarwar, M., Zada, M. B., Manro, S., Common fixed point theorems for weakly compatible mappings in complex valued metric spaces, Bulletin of the International Mathematical Virtual Institute, 7, (2017), 11-21.

[32] Sarwar, M., Humaira, H., Li, T., Fuzzy Fixed point results and applications to ordinary fuzzy differential equations in complex valued metric spaces, Hacettepe Journal of Mathematics and Statistics, 48(6) (2019), 1712-1728. https://doi.org/10.15672/HJMS.2018.633.

[33] Shatanawi, W., Norani, J. A., Alsamir, H., Kutbi, M. A., Some common fixed points of multivalued mappings on complex-valued metric spaces with homotopy result, Journal of Nonlinear Sciences and Applications, 10 (2017), 3381-3396. https://doi.org/10.22436/JNSA.010.07.02.

[34] Sintunavarat, W., Kumam, P., Generalized common fixed point theorems in complex valued metric spaces and applications, Journal of Inequalities and Applications, 2012 (2012), article 84. https://doi.org/10.1186/1029-242X-2012-84.

[35] Sunanda, R., Patil, J., Salunke, N., Common fixed point theorems in complex valued rectangular metric spaces, South Asian Journal of Mathematics, 6(1) (2016), 10-23. 